\title{
WARUM HABEN DIE REGENFAKTOREN SO HÄUFIG IN KLIMATOLOGIE UND BODENKUNDE VERSAGT?
}

\author{
Paul Schaufelberger
}

\section{EINLEITUNG}

In der gemäßigten Zone (7) stellt man sich - wohl seit uralter Zeit - gerne die Tropen folgendermaßen vor:

1. Temperatur :

2. Klima :

3. Vegetation:

\section{heiß}

trocken

Steppe

aufsteigend

wechselfeucht

Savanne

immerfeucht

Regenzuald

abzürts versickernd

Natürlich gibt es in den Tropen trockene, wechselfeuchte und immerfeuchte Klimate, sowie Steppen, Savannen und Wälder (aber auch Salzsteppen, zeitweise vernäßte Anmoore und ständig vernäßte bis überschwemmte Niedermoore) und Böden mit den verschiedenen Wasserbewegungen. Böden mit allen denkbaren Perkolationsrichtungen findet man beispielsweise im ariden Klima, so daß dieses wohl kaum die Ursache der Wasserbewegung im Boden sein kann. Natürlich sind die tropischen Küsten heiß, was aber nicht auf die Hochgebirge und Gletscherregion in Aequatornähe zutrifft.

Diese Tatsachen sind in der gemäßigten Zone kein Geheimnis, aber offenbar finden sie nicht immer die volle Berücksichtigung.

\section{KLIMA UND VEGETATION}

E. Scherf (20) fand im semihumiden Klima Schlesiens nebeneinander: Podsol, Braunerde, Tschernosem und anmoorige Böden. Da in der offlziellen Bodenkunde Podsol, Braunerde und Tschernosem als Klimaböden perhumider, humider und semihumider Bereiche gelten, so mußte diese Feststellung überraschen, denn darnach müßten in ein und demselben Klima sich drei «Klimaböden» bilden. Es kann daher nicht überraschen, daß diese Tatsache in der Folge gerne benützt wurde, um zu «beweisen », daß die Regenfaktoren nicht stimmen können.

Scherf gibt für das Nebeneinandervorkommen dieser Bodentypen nachstehende Erklärung: "Also nicht das Luftklima allein ist bestimmend für die Tschernosembildung in diesen Gebieten, sondern (und in viel höherem Maße) das Bodenklima. Welchem unter den verschiedenen, stets zusammen wirksamen bodenklimatischen Faktoren die Hauptrolle einzuräumen sei, ob es die Vegetation ist (HoHenheim und SchalOw) oder das Relief (ORTH) oder die Wasserundurchlässigkeit des Untergrundes und der Bodenkrume, zusammen mit deren Karbonatgehalte (Jentzsch und SCHERF), müssen weitere Untersuchungen entscheiden».

Mit dieser Frage haben sich verschiedene Autoren befaßt. H. Stremme $(21,22)$ hat beispielsweise gezeigt, wie sich die Böden nach Profil und natürlicher Vegetation in sechs Bodenformen klassieren lassen. C. Troll (24) sagte in einem Vortrag über das Pflanzenkleid der Tropen: «Der Anteil von Gras-und Holzpflanzen in den tropisch wechselfeuchten Klimaten ist nämlich nicht abhängig vom Klima, sondern von anderen ökologischen Faktoren, edaphischen, biotischen und anthropogenen, die bekanntlich auf kleinem Raume außerordentlich vielfältig wechseln können und ein buntes Vegetationsbild erzeugen». TROLL benützt für die tropischen Vegetationstypen den Vorschlag von JAEGER, der sechs Formen unterscheidet. Der Verfasser (9) fand im ariden Klima Kolumbiens Wald, Steppen, Salzsteppen und bringt diese Vegetation mit der Wasserbewegung im Boden in Verbindung. Nach dieser (16) unterscheidet er sechs Bodenklassen und definiert sie durch die Perkolationsrichtung, d. h. durch das geologische Substrat. Zwischen diesen verschiedenen Vorschlägen finden wir folgende Beziehungen. 


\section{Tabelle 1. Boden und Vegetationstyp}

\begin{tabular}{|c|c|c|c|}
\hline SCHER & STREMME & TROLL/J JGER & SCHAUFELBERGER \\
\hline 1. Braunerde, Podsol & Waldboden & Wald & endoperkolativer Boden \\
\hline 2. Tschernosem & Mineralnaßboden & Feuchtsavanne & peri-endoperkolativer Boden \\
\hline - & Steppenboden & Trockensavanne & amphiperkolativer Boden \\
\hline 一 & Salzboden & Dornsavanne & exoperkolativer Boden \\
\hline 5. Anmoor & Anmoor & Halbwüste & peri-exoperkolativer Boden \\
\hline 一 & Moor & Wüste & periperkolatives Moor \\
\hline
\end{tabular}

Daß Wald, Savanne und Steppe edaphisch bedingt sind, geht aus allen vier Vorschlägen eindeutig hervor. Dagegen sind Dornsavanne, Halbwüste und Wüste klimabedingte Vegetationen des ariden Klimas. Daß Steppe und Salzsteppe, Mineralnaßboden und Anmoor häufig in der Bodenkunde nicht auseinander gehalten werden, ist eine alte Tatsache, aber auch, daß gerade die Vegetation sehr leicht auf den Salzgehalt reagiert. Auffallend aber ist, daß im Vegetationstypeninventar von TroLL und JAEGER die in allen Klimaten vorkommenden Niedermoore fehlen.

Wald, Mineralnaßboden (Wiesen und Savannen) und Moore finden wir in allen Klimaten, sie müssen daher edaphisch bedingt sein, ihre Verbreitung kann daher nicht durch bestimmte Regenfaktoren begrenzt sein. Anmoore kennt man aus ariden bis semihumiden Klimaten, Steppen aus ariden und semiariden, während Salzsteppen in der Hauptsache auf aride Bereiche beschränkt sind. Da aber in diesen Klimaten noch Wald, Mineralnaßböden und Anmoore vorkommen, so ist keiner dieser Vegetationstypen durch einen bestimmten Klimafaktor begrenzt. Will man mit den Regenfaktoren allein den Vegetationstyp bestimmen, so müssen sie versagen, weil sich mit einem Klimafaktor die Grenzen geologisch bestimmter Bodenklassen nicht erkennen lassen.

\section{RELIEF, BODENKLASSE UND VEGETATIONSTYP}

R. LANG (5) begann seine bodenkundlichen Forschungen im Schwarzwald und erkannte dort verschiedene Bodenformen, die er mit dem Klima in Verbindung brachte. Seinen Regenfaktor formulierte er nach seiner Rückkehr aus den Tropen und bezeichnete unglücklicherweise die fünf Klimaböden als Bleicherde (Podsol); Schwarzerde; Braunerde; Laterit; Rot- und Gelberde; Böden arider Gebiete. Es war und ist ein Irrtum der Bodenkunde, daß sie den Bodenfarben zu große Bedeutung zulegte und teilweise noch heute zulegt, worauf zahlreiche Autoren aufmerksam gemacht haben.

H. Jenny (3) schrieb 1928 über die Böden der Schweiz: «Die Böden des Juras, der kalkreichen Voralpen und Alpen (inkl. der Schiefer) sind in eine große Gruppe «starke Umlagerung der Karbonate» zusammengefaßt worden. Treffender wäre zu sagen: starke Auswaschung der Karbonate. Die Böden sind keine echten Klimaböden, da sie an kalkführende Schichten gebunden sind. «Hier sagt JENNY zutreffend, daß Böden auf kalkführenden Gesteinen keine echten Klimaböden seien. Das ist eine negative Feststellung, denn damit ist nur gesagt, welche Böden nicht als Klimaböden in Frage kommen, aber nicht, welches die echten Klimaböden sind.

Er bestätigt die Beobachtung Scherf's, daß sich je nach dem Karbonatgehalt im selben Klima verschiedene Böden bilden. Seine Braunerde ist daher kein echter Klimaboden, als solcher kommt sein Podsol in Frage, aber dieser dürfte kaum identisch sein mit dem Klimaboden der perhumiden Gebiete, der mit demselben Namen beschrieben ist. Seine Arbeit zeigt deutlich, daß die damalige Bodenkunde die Klimaböden als solche nicht kannte.

Der Verfasser $(10,11,15)$ fand an den Hängen der kolumbianischen Anden in jedem Klima ebenfalls jeweils zwei Bodentypen. Den einen bezeichnet er als Klimasol und bringt ihn mit dem Klima in Verbindung, den andern als Lithosol, der den Einfluß des Muttergesteins zeigt. Diese Böden findet man sowohl auf kalkführenden wie basenreichen silikatischen Gesteinen. Seine Untersuchungen ergaben, da $\beta$ die Klimasols arm, die Lithosols reich an austauschbaren Basen sind (7, 8, 11, 14). Im Schwarz- 
wald, im Jura und den Alpen der Schweiz, und in den Anden Kolumbiens bilden sich je nach dem Muttergestein und dem Alter der Bodenbildung zwei Bodentypen: die basenreichen Lithosols, die nach JENNY (3) keine echten Klimaböden sind, und die Klimasols, die JeNNY nicht kennt und die mit den von LANG genannten Bodentypen nicht identisch sind. Es sind aber auch nicht Sierosem, Kastanienboden, Tschernosem und Braunerde, wie die offizielle Bodenkunde behauptet. Offenbar bilden sie sich im Laufe der Zeit durch Anpassung ans Klima aus den genannten Böden.

Kennt man nun bei Hangböden, d.h. normal drainierten oder endoperkolativen Waldböden den Gehalt an austauschbaren Basen und den Regenfaktor, dann läßt sich eindeutig entscheiden, welcher Klima- oder Gesteinsbodentyp vorliegt, weil das Relief nur die Bildung von Waldböden erlaubt.

Andere Verhältnisse treffen wir in Ebenen, wo neben den Waldböden sich auch die Hydrosols bilden, an deren Entwicklung die Hydrologie irgend in einer Form beteiligt ist. Im Norden finden wir die Tundrenböden, im gemäßigten Klima die Gleiböden und in den Tropen die Schwarzerden, die baumfeindlich sind. Sie verteilen sich auf Mineralnaßböden, Steppen, Salzsteppen, Anmoore und Moore, die geologisch durch die Wasserbewegung bedingt sind. Hier muß man erst die Bodenklasse bestimmen, wozu sich die Regenfaktoren als Klimafaktoren natürlich nicht eignen.

\section{DIE KLIMABODENTYPEN}

In seiner viel zitierten Arbeit über Klima und Klimaböden, beschreibt H. Jen Ny (4) Böden der verschiedenen Klimate der USA. Nun haben wir aber bereits gesehen, daß als echte Klimaböden nur basenarme Waldböden in Frage kommen, deswegen müssen wir den Podsol des perhumiden Klimas ausschalten, weil es sich um ein Hydrosol handelt. Dann sagt Jenny (3) 1928, daß Böden auf kalkführenden Gesteinen, mit starker Auswaschung von $\mathrm{Ca}$, keine echten Klimaböden seien. Dazu gehören folgende Böden seiner Arbeit von 1929: Wüstenboden, Kastanienboden I und II, Tschernosem I, Brauner Waldboden und Waldsteppenboden II, wie wir früher gezeigt haben (18). Damit erbringt Jenny den Beweis, daß die Klimaböden der offiziellen Bodenkunde keine echten Klimaböden sind. Weiter zeigt dieser Autor, daß der Tschernosem sich bei Regenfaktoren zwischen 40 und 150 bildet, was ebenfalls unterstreicht, daß der Tschernosem kein Klimaboden sein kann. Eigene Untersuchungen $(8,14)$ bestätigen diese Feststellung.

Als echte Klimaböden kommen dann nur noch folgende Böden in Frage. Tab. 2.

Tab. 2. Echte Klimaböden der USA

$\begin{array}{lccc}\text { Boden (JENNY) } & \text { Regenfaktor } & \text { Auswaschungsfaktor Bodentyp } \\ \text { Tschernosem II } & 69 & + \text { si }+ \text { c +alk } & \text { Humussol } \\ \text { Rot- und Gelberde II } & 82 & + \text { si+c+alk } & \text { Humussol } \\ \text { Waldsteppenboden I } & 100 & + \text { si+c+alk } & \text { Humussol } \\ \text { Rot- und Gelberde II } & 115 & + \text { si-c+alk } & \text { Waldsol }\end{array}$

In Kolumbien, sowie anderen Gebieten der Tropen und der gemäßigten Zone wurden bei den normal drainierten, endoperkolativen Waldböden bei den verschiedenen Regenfaktoren folgende Auswaschungsfaktoren gefunden. Tab. 3.

Tab: 3. Auswaschungsțakoren der Waldböden

\begin{tabular}{|c|c|c|c|}
\hline Regenfaktor & basenarme Klimasols & basenreich & Lith \\
\hline unter $\quad 40$ & $+s i-c-a l k$ & + si-c+alk & $+\mathrm{si}-\mathrm{c}-\mathrm{alk}$ \\
\hline $40-60$ & $+\mathrm{si}+\mathrm{c}-\mathrm{alk}$ & $+\mathrm{si}-\mathrm{c}+\mathrm{alk}$ & $+\mathrm{si}+\mathrm{c}-\mathrm{alk}$ \\
\hline $60-100$ & + sitctalk & $+\mathrm{si}-\mathrm{c}+\mathrm{alk}$ & $+\mathrm{si}+\mathrm{c}-\mathrm{alk}$ \\
\hline $100-160$ & $+s i-c+a l k$ & $+\mathrm{si}-\mathrm{c}+\mathrm{alk}$ & \\
\hline über 160 & $+\mathrm{si}-\mathrm{c}-\mathrm{alk}$ & + si-c+alk & \\
\hline
\end{tabular}

* Bei den Ausnahmen handelt es sich um junge, unreife Gesteinsböden oder solche, die gerade im Begriffe sind zu den Klimasols zu degradieren; sie sind selten. 
JENNY's echte Klimaböden zeigen bei denselben Regenfaktoren genau dieselben Auswaschungsfaktoren wie die Klimasols der Tabelle 3. Dagegen zeigt die Tabelle 2, daß man in den USA im Jahre 1929 die Klimaböden arider, semiarider und perhumider Bereiche nicht kannte.

\section{REGENFAKTOREN UND KLIMA}

Jenny (4), Tamm (23), Gracanin (1), Troll (24) und Verfasser (17) zeigen, daß zur Beurteilung des Klimas allermindestens zwei von einander unabhängige Faktoren notwendig sind: Jahrestemperatur und Befeuchtung. Die ältere klimatologische Literatur definiert die Befeuchtung durch Niederschlag und Verdunstung. LANG definiert sie als Regenfaktor $=$ Niederschlag durch Temperatur. Davon abgeleitet sind der Ariditätsfaktor und die Faktoren von Thornthwaite. Bei Verwendung von monatlichen Klimafaktoren werden außer denjenigen von Thornthwaite, die Ariditätsindices und die Regenfaktoren benützt. Damit ist die Definition der Befeuchtung durch Niederschlag und Verdunstung praktisch bedeutungslos geworden.

Offenbar wird bei der Verwendung von Jahrestemperatur und Befeuchtung der Einflu $\beta$ der Zone als gegeben oder konstant vorausgesetzt. Für große Gebiete sind, wie früher gezeigt worden ist (19), Zone, Jahrestemperatur und Befeuchtung (Regenfaktor) zu berücksichtigen, einer Auffassung, der Gracanin (2) seine Zustimmung gegeben hat. Daraus ergibt sich, daß man mit dem Regenfaktor allein kein Klima genügend charakterisieren kann.

\section{ZUSAMMENFASSUNG}

1. Bei optimalen Bodenbildungsbedingungen entwickeln sich bei den verschiedenen Regenfaktoren in allen Zonen dieselben Klimabodentypen. Aber man darf daraus nicht schließen, daß sich bei bestimmten Regenfaktoren nur die Klimabodentypen bilden. Mit den Regenfaktoren kann man die Bodentypen daher nicht klassieren.

2. Die edaphisch bedingten Vegetationstypen sind: Wald, Savanne (Feuchtsavanne), Steppe (Trockensavanne), Salzsteppe, Anmoor und Moor. Ihre geographische Verbreitung ist durch das geologische Substrat bestimmt, also unabhängig vom Klima. Jeder Versuch mit dem Regenfaktor Schlüsse auf den Vegetationstyp zu ziehen, müssen darum scheitern.

3. Die Regenfaktoren definieren die Befeuchtung: arid, semiarid, semihumid, humid und perhumid.

4. Das Klima ist durch Zone, Jahrestemperatur und Befeuchtung (Regenfaktor) zu charakterisieren.

5. Will man mit dem Regenfaktor mehr als die Befeuchtung bestimmen, so versagen sie, weil sie am unrichtigen Platz angewendet werden.

\section{BIBLIOGRAPHIE}

1. Gracanin, M. 1950 : Monthly rain-factors and their significance for pedological investigations. Isdavacki Zavod Jugoslavenske Akadeie 22. - 2. Gracanin, M. 1957 : Briefliche Mitteilung. 3. Jenny, H. 1928 : Bemerkungen zur Bodentypenkarte der Schweiz. Landwirtschaftl. Jahrb. d. Schweiz 42/3. - 4. Jenny, H. 1929: Klima und Klimabodentypen in Europa und den Vereinigten Staaten von Nordamerika. Bodenkundliche Forschungen I/3. - 5. LANG, R. 1915: Versuch einer exakten Klassifikation der Böden in klimatischer und geologischer Hinsicht. Int. Mitt. f. Bodenkunde. 6. Schaufelberger, P. 1950 : Wie verläuft die Gesteinsverwitterung und Bodenbildung in den Tropen, insbesondere in Kolumbien? Schweiz. Min. und Petr. Mitt. 30 2. - 7. Schaufelberger, P. 1952 a: Zur Gliederung des tropischen Klimas. Geogr. Helv. VII/2. - 8. Schaufelberger, P. 1952 b: Gibt es in den Tropen Tschernosem? Zeitschr. f. Pflanzenern., Düngung und Bodenkunde 57/2. 9. Schaufelberger, P. 1952 c : Zur Klassifikation der ariden tropischen Böden. Experimentia VIII/9. 10. Schaufelberger, P. 1952 d: Die Klimabodentypen des tropischen Kolumbiens. Vierteljahrsschr. d. Naturf. Ges. in Zürich 97, p. 92-114. - 11. Schaufelberger, P. 1953 a: Die nicht zonalen Bodentypen des tropischen Kolumbiens. Ibid. 98, p. 88-108. - 12. Schaufelberger, P. 1953 b : Tropische Verwitterung und Bodenbildung über Andesit und Diorit. Schweiz. Min. u. Petr. Mitt. 33/1. - 13. Schaufelberger, P. 1954 a: Verwitterung und Bodenbildung auf basischen Eruptivgesteinen. Ibid. 34/2. - 14. Schaufelberger, P. 1954 b: Ist der Tschernosem ein zonaler Klima- 
bodentyp? Experimentia X/6. - 15. Schaufelberger, P. 1955 a: Eignen sich die Regenfaktoren Lang's zur exakten Klassifikation der tropischen Böden? Peterm. Geograph. Mitt. Heft 2 . 16. Schaufelberger, P. 1955 b: Zur Systematik der tropischen Böden. Vierteljahrsschr. d. Naturf. Ges. in Zürich 100, p. 131-142. - 17. Schaufelberger, P. 1955 c: Vierzig Jahre Regenfaktor. Ibid. 194-201. - 18. Schaufelberger, P. 1956: Kritische Betrachtungen der Bodenklassifikationen. Schweiz. Min. u. Petr. Mitt. 36/2. - 19. Schaufelberger, P. 1957 : Zur Systematik des Tropenklimas. Geogr. Helv. 12/1. - 20. Scherf, E. 1930 : Über die Rivalität der boden- und luftklimatischen Faktoren bei der Bodentypenbildung. Annalibus Institutu Regii Hungarici Geologici 24. 21. Stremme, H. 1926: Grundzüge der praktischen Bodenkunde. Berlin. - 22. Stremme, H. 1949: Die Böden der Deutschen Demokratischen Republik. Berlin. - 23. Taмm, O. 1932: Die Böden Schwedens. Ref. in D. Ern. d. Pflanze 28/17. - 24. Troll, C. 1952 : Das Pflanzenkleid der Tropen in seiner Abhängigkeit vom Klima, Boden und Mensch. Festvortrag, gehalten auf dem Deutschen Geographentag in Frankfurt a. M. am 13. Mai 1951, veröffentlicht in Tagungsberichte und wissenschaftliche Abhandlungen, Remagen.

\section{LES FACTEURS DE PLUVIOSITÉ EN CLIMATOLOGIE ET PÉDOLOGIE}

A l'aide des facteurs de pluviosité les types de sol ne peuvent pas être classifiés. Les types de végétation de cause édaphique sont: forêt, savanne, steppe, steppe saline et marais. Leur répartition géographique est déterminée par le substrait géologique, c'est-à-dire indépendant du clima. Tout essai de déterminer les types de végétation d'après les facteurs de pluviosité devra pour cette cause échouer. Les facteurs de pluviosité déterminent le degré d’humidité : arid, semiarid, humid et perhumid. Le clima est à caractériser d'après la zone, la température annuelle et le facteur de pluviosité. L'intension de vouloir définir avec les facteurs de pluviosité autre sujet que le de degré d'humidité échouera.

\section{DER KONGRESS DES INTERNATIONALEN GEOGRAPHIE- LEHRER-VERBANDES IN GRENOBLE}

\section{Georg Sprecher}

Vom 26.-31. August 1957 fand in Grenoble der dritte Kongreß dieses lose organisierten Verbandes statt. Die Durchführung der Veranstaltung hatte das in Fachkreisen angesehene «Institut de Géographie Alpine» in Grenoble unter Prof. P. Veyret-Verner übernommen, wobei auch Frau Prof. Veyret-Verner wesentlich zum Gelingen des Kongresses beitrug. Mit Umsicht war alles wohl vorbereitet. Der größte Teil der Teilnehmer logierte in dem erst einjährigen «Internat du Lycée des Jeunes Filles», einem neungeschossigen Bau am Stadtrande von Grenoble, wo man auch aufs beste verpflegt wurde. Wenn Pünktlichkeit eine Tugend der Könige sein soll, so wurde man in Grenoble zu Königen erzogen und fürstlich geführt. Tagungsort und Programm lockten etwa doppelt soviel Teilnehmer wie 1954 an, nämlich 300 Damen und Herren aus 20 Ländern und von allen Kontinenten. Zahlenmäßig am stärksten waren neben Frankreich Italien und Großbritannien vertreten. Die Schweizer Delegation bestand aus fünf Herren.

Die sechs Kongreßtage verliefen sehr abwechslungsreich, und es war neben den Vorträgen und Exkursionen noch genügend Zeit, um Diskussionen anbahnen zu können.

Nach den Begrüßungsansprachen vermittelte der erste Tag durch den überlegenen Vortrag von Prof. Veyret über die Westalpen im allgemeinen und über Grenoble im besonderen eine ausgezeichnete Einführung in Tatsachen und Probleme der umgebenden Region. Die nachmittägliche Exkursion auf die «Bastille» rundete den Eindruck über Stadt und Land ab.

Einige Ausführungen über Grenoble seien nachfolgend zusammengefaßt:

Der natürliche Rahmen von Grenoble ist die weite Ebene, umgeben im Osten durch die kristalline Kette der Belledonne, im Westen durch die beiden voralpinen Kalkmassive der Grande Chartreuse und des Vercors. Die Stadt liegt in einer großen Talkreuzung, die ebenso eine Verbindung ins Vorderland und nach Italien, wie nach Norden und Süden herstellt. Hier vereinigt sich der wilde Drac mit der Isère und 